\title{
Medical Acupuncture for Intracerebral Bleed: A Case History
}

\author{
Allen McDaniels, MD
}

\begin{abstract}
Background: A 75-year-old woman with a history of atrial fibrillation, anticoagulant therapy, and grand mal seizures sustained a right lateral ventricular intracerebral bleed. After being unconscious for 1 week in the intensive care unit of a general hospital, she spent another 2 weeks in intensive care before being transferred to a long-term acute care hospital for a month and to a comprehensive rehabilitation hospital for 3 weeks.

Objective: To describe an important role for medical acupuncture in treating stroke and complementing standard medical management.

Methods: Medical acupuncture was used to reverse left hemiparesis, improve mental awareness and focus, and hasten the course of recovery.

Conclusion: Medical acupuncture should have an important role in the acute and subacute phases of treatment of hemorrhagic stroke.

Key Words: Auricular Acupuncture (AA), Chinese Scalp Acupuncture (CSA), Intracerebral Bleed, Medical Acupuncture, Stroke

\section{INTRODUCTION}

A CUPUNCTURE FOR THE TREATMENT of stroke, whether embolic or hemorrhagic, has been proposed by several sources (Hao JJ, Helms JM. Workshop: Chinese Scalp Acupuncture. Berkeley: Helms Medical Institute). ${ }^{1,2}$ This article provides a clinical justification for acupuncture's use as an important therapeutic tool in the treatment of hemorrhagic stroke.

Increased use of anticoagulants in recent years correlates with an increased frequency of warfarin-related intracerebral hemorrhage. A high early mortality rate of $50 \%$ has remained stable for decades. ${ }^{3,4}$

\section{CASE REPORT}

The patient, a 75-year-old woman, had a decades-long history of atrial fibrillation that began in 1977 after her

mother's death. She also had had a grand mal seizure disorder since adolescence. Her atrial fibrillation had been treated with oral warfarin (Coumadin, Bristol-Myers Squibb, New York, NY), monitored early with prothrombin time and partial thromboplastin time testing, and later with international normalized ratio (INR). Two weeks before the stroke, the patient's INR was 2.2 (therapeutic level, 2.0-3.0). In addition, the patient received an oral $\beta$-blocker, atenolol (Tenormin, AstraZeneca, Wilmington, DE), to control heart rate. Blood pressure had always been within normal range. Seizures were well controlled with phenytoin (Dilantin, Pfizer, New York, NY), but compliance with this medication was erratic. She had not had a witnessed seizure in at least 15 years.

The patient had been in good health until approximately 2 years before stroke, when she experienced extreme fatigue, lassitude, ennui, and mild exogenous depression, which significantly limited her physical, mental, and social activities. Cardiac, neurologic, and rheumatologic work-ups
\end{abstract}

Helms Medical Institute, San Pedro, CA. 
were inconclusive. No additional therapy was ordered. She had nearly recovered to premorbid functional levels when the stroke occurred.

On day 0 , the patient experienced severe gait instability, falling to the left, urinary frequency, and increasing stupor over several hours. No lateralizing signs, save the falling to the left, were noted. Paramedics transported her to a nearby general hospital; by then the patient was comatose with left hemiparesis. The Glasgow coma score was $6(1+1+4)$. Computed tomography revealed a large right lateral ventricular capillary bleed originating at the level of the basal ganglia. Mild-to-moderate deviation of the falx cerebri to the left and cerebral edema were noted. Supportive treatment in the hospital's medical intensive care unit (ICU) for reversal of anticoagulation, cerebral edema, unstable blood pressure (BP), coma, and flaccid hemiparesis followed.

In Traditional Chinese Medicine stroke is due to internal Wind with Phlegm clogging the mind's orifices. This is called Wind-Stroke. Internal Wind always gives rise to a Liver disharmony. The main manifestations of internal Wind are tremors, tics, severe dizziness, vertigo, and numbness. In extreme cases there are convulsions, unconsciousness, opisthotonos, and hemiplegia. ${ }^{5}$

The patient remained comatose (but slightly responsive to commands with hand pressure) and hemiparetic. On day 4, a family friend suggested playing music from her young adult life, the early 1950s, that he knew she loved. Through a computer and earphones, her husband played tunes of the Four Freshmen, Billie Holliday, and Hoagie Carmichael. Within several minutes the patient became more responsive to simple commands with firmer right hand pressure. On day 5, Chopin nocturnes and Mozart's Clarinet Concerto were added, with similar responses to commands. Music also helped to stabilize her BP.

On day 7, the patient opened her eyes and retreated from coma. A week later, computed tomography revealed significantly less cerebral edema and blood in the lateral ventricle. On day 15 she exhibited spontaneous movement of her left leg and arm. This was reinforced by a medical acupuncturist deep needling left KB 1 and KB 2, which encouraged more movement of the left limbs, with the leg responding more than the arm. (The KB points$\mathrm{KB} 1$ in the forearm and KB 2 in the lower leg-attributable to Joseph Y. Wong's neuroanatomical work, ${ }^{6}$ are interosseus points that influence the sympathetic connections there.)

On day 17, the patient was transferred to a long-term intensive care facility nearby because the general hospital did not have a step-down unit for stroke patients. At the new facility, it was much easier to administer acupuncture without the intense scrutiny of a level 1 ICU. Chinese Scalp Acupuncture (CSA), administered by the medical acupuncturist, began immediately. Needling and manually stimulating the right upper fifth and middle two fifths of the motor strip almost immediately produced very active movement of the left leg and arm. The next day the patient could actively move her left leg, but there was little movement of her left arm. CSA was repeated the next 2 days, with increasing active movement of the left arm and leg and increased mental alertness for up to 4 hours. On day 21 she was transferred out of the ICU to a monitored ward.

On day 22, the medical acupuncturist administered auricular therapy to the left brain point and motor area for arm (posterior surface of ear). He also strongly stimulated left KB 1. The patient reported feeling the acupuncture treatment for the first time during her recovery. Administering KB 1 also decreased left arm flaccidity.

Over the next several days, ear points (left and right), CSA, KB points, and trigger point deactivation were administered at least once daily, sometimes more often depending on the patient's stamina. A menu of ear points included Brain, Hypothalamus, Basal Ganglion, and motor points of arm and leg. CSA therapy involved right upper fifth and middle two fifths of the motor strip plus foot motor sensory with needles in place for 2-3 hours or more. More voluntary movement of the left side, increased mental alertness, more attempts at speaking (although tracheostomy tube was in place), and beginning attempts at fine motor movement (writing) resulted.

By day 31, the patient began to have an intermittent choreiform tremor of her right arm. To her usual CSA treatment, the chorea/tremor strip was added bilaterally. This quickly resolved the tremor. Ear points Adrenal C, Adrenal F, Autonomic, Brain, Adrenocorticotropic Hormone, Master Cerebral, and Endocrine as a general treatment for stroke. ${ }^{7}$ Minor Occipital Nerve and Occiput were added in groups of 5 or 6 ear points using barbed tabs (Pyonex, Seirin, Weymouth, MA; by now the patient had developed an intolerance for needles in her ears) and left in place for a few days. Added to this were Hypothalamus, Amygdala, Hippocampus, Master Cerebral, Point Zero, and Shen Men (auricular trauma protocol) plus the motor points for leg and arm with multiple tabs. The result was improved movement of the left side and alertness with better mental focus. With improved alertness and focus the patient became very talkative.

At this stage of the patient's recovery, Windows of the Sky points to reconnect head and body after brain trauma were palpated and tender ones needled. ${ }^{8}$ This treatment was repeated several times a week during the next month and after the patient returned home, to very good effect.

Extensive Curious Meridian therapy was added by using Du Mo (for central nervous system) initially, then Chong $\mathrm{Mo} / \mathrm{Du} \mathrm{Mo}$ and Dai Mo/Du Mo, which enhanced the other acupuncture modalities.

The patient's self-awareness returned on day 48 with a flood of emotion upon realizing that she had had a stroke 
and was experiencing related disabilities. CSA with Two Dragons (BL 4 to BL 7 threaded anterior to posterior bilaterally) and Temporal Fan (3 needles diagonally threaded in the scalp over the temporal lobe) plus GV 20, 22, 24, and 24.5 and Si Shen Cong tempered emotions and provided improved mental focus.

On day 49, she was transferred to a comprehensive rehabilitation hospital for rigorous physical and occupational therapy. Because the patients was not yet able to pass the swallowing test for approval to eat more solid food, the medical acupuncturist began treatment with throat points (LU/LI Distinct Meridian with focus at ST 9, plus extra point Zengyin at each end of the hyoid bone). This enabled the patient to eat solid food, such as puddings and pureed foods.

On day 51, she could walk 25-30 feet with assistance, was well oriented, and could converse well. A week later the assisted walking increased to near 100 feet. Transfers from bed to wheelchair or from wheelchair to toilet remained difficult.

The combination of stroke recovery, hospital food, and a percutaneous endoscopic gastrostomy in place affected the patient's appetite, which would wax and wane. Improved mental awareness with needling the GV line from 18 to 24.5 and Two Dragons helped her appetite the most. Adding electricity at $15 \mathrm{~Hz}$ between GV 20 and GV 24.5 greatly improved her mood as she continued to come to terms with her medical predicament.

On day 66 after the stroke, CSA with frontal triangle and right praxia area improved alertness, social interaction, and purposeful eating. Her prestroke personality re-emerged. The patient was discharged to home and the care of her family on day 69.

\section{DISCUSSION}

In summary, this case history of treatment for recovery from a large intracerebral bleed demonstrates the effectiveness of medical acupuncture as an important therapeutic modality for recovery of function. Various modes of acupuncture were used depending on the time frame of recovery and the deficits manifest. KB points and CSA were the workhorses for addressing the neurologic lesions. Ear points, both sensory and motor, came next in importance, along with Windows of the Sky and Du Mo/Chong Mo. The latter two treatment approaches were used for both enhancement of $\mathrm{KB}$ points and CSA and for symptomatic treatment.

Regarding KB points and their effect on the cerebral cortex, clinical observations indicate an intimate connection between the two, thus the effect in this patient of KB needling moving a flaccid limb, possibly through a spinal reflex arc, but soon facilitating voluntary motor function. $\mathrm{KB}$ points influence the neurovascular structures that run in the interosseus membrane, particularly the sympathetic connections. The presynaptic fibers and postsynaptic sympathetic fibers carry visceral sensory afferent fibers. Autonomic fibers also invest the arterioles. In addition, there is autonomic representation on the cerebral cortex, where higher integration of the autonomic nervous system may occur. Because the autonomic nerves are considered motor nerves, autonomic and motor cortical function often overlap. $^{9}$

$\mathrm{KB} 1$ of the upper extremity receives the posterior interosseus nerve from the deep branch of the radial nerve. The median nerve sends the anterior interosseus nerve and smaller branches to the interosseus membrane. KB 2 of the lower extremity receives branches of the deep peroneal nerve. $^{10}$

The practice of auricular acupuncture too often focuses on the sensory (outer) surface and neglects the motor (inner) surface. This patient had a hemiparesis, which required a restoration of motor function. Needling the ear's motor surface for upper and lower extremities proved effective as part of this restoration.

\section{CONCLUSION}

Medical acupuncture for treating stroke patients requires further research to determine its scope of usefulness. However, if this case history is a typical example of an intracerebral bleed, medical acupuncture should be initiated as soon after insult as neurologic deficits clinically manifest themselves. Not to do so could be overlooking a potentially valuable therapeutic approach for treatment and recovery.

\section{AUTHOR DISCLOSURE STATEMENT}

No competing financial interests exist.

\section{REFERENCES}

1. Yamamoto Y, Yamamoto H. Yamamoto New Scalp Acupuncture. Tokyo: Axel Springer Japan Publishing; 1998:192193.

2. Hao JJ, Hao LL. Chinese Scalp Acupuncture. Boulder, CO: Blue Poppy Press; 2011:87-90.

3. Aguilar MI, Hart RG, Kase CS, et al. Treatment of warfarinassociated intracerebral hemorrhage: literature review and expert opinion. Mayo Clin Proc. 2007;82(1):82-92.

4. Rosand J, Eckman MH, Knudsen KA, Singer DE, Greenberg SM. The effect of warfarin and intensity of anticoagulation on outcome of intracerebral hemorrhage. Arch Intern Med. 2004; 164(8):880-884.

5. Maciocia G. The Foundations of Chinese Medicine. Edinburgh: Churchill Livingstone; 1989:196,296. 
6. Wong JY. A Manual of Neuro-Anatomical Acupuncture. Vol. 1. Toronto: Joseph Y. Wong; 1999.

7. Oleson T. Auriculotherapy Manual: Chinese and Western Systems of Ear Acupuncture, 2nd ed. Los Angeles: Health Care Alternatives; 1996:192.

8. Helms JM. Acupuncture Energetics. Berkeley: Medical Acupuncture Publishers; 1995:444-447.

9. Chusid JG. Correlative Neuroanatomy \& Functional Neurology, $18^{\text {th }}$ ed. Los Altos: Lange Medical Publications; 1982: 140, 149, 150.
10. Netter FH. Atlas of Human Anatomy. East Hanover, NJ: Novartis; 1989: Plates 419, 422, 491.

Address correspondence to: Allen McDaniels, MD Helms Medical Institute 732 West Ninth Street, Suite 200 San Pedro, CA 90731

E-mail: qmcduck@pacbell.net 